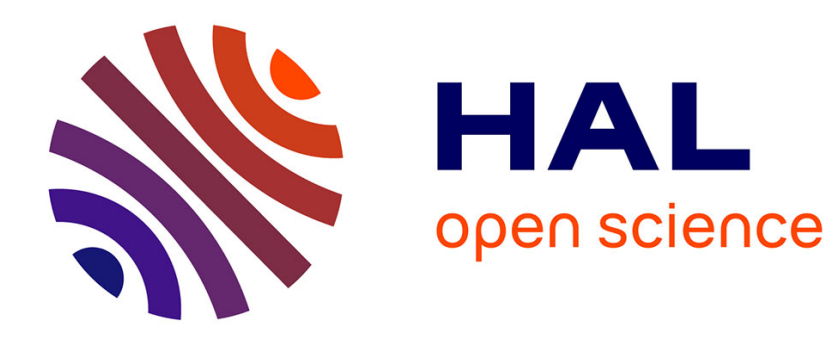

\title{
Conduction inverse par éléments de frontière. Cas stationnaire
}

\author{
R. Pasquetti, C. Le Nilliot
}

\section{To cite this version:}

R. Pasquetti, C. Le Nilliot. Conduction inverse par éléments de frontière. Cas stationnaire. Revue de Physique Appliquée, 1990, 25 (1), pp.99-107. 10.1051/rphysap:0199000250109900 jpa-00246167

\section{HAL Id: jpa-00246167 https://hal.science/jpa-00246167}

Submitted on 1 Jan 1990

HAL is a multi-disciplinary open access archive for the deposit and dissemination of scientific research documents, whether they are published or not. The documents may come from teaching and research institutions in France or abroad, or from public or private research centers.
L'archive ouverte pluridisciplinaire HAL, est destinée au dépôt et à la diffusion de documents scientifiques de niveau recherche, publiés ou non, émanant des établissements d'enseignement et de recherche français ou étrangers, des laboratoires publics ou privés. 


\title{
Conduction inverse par éléments de frontière. Cas stationnaire
}

\author{
R. Pasquetti et C. Le Nilliot \\ Laboratoire Systèmes Energétiques et Transferts Thermiques, U.A. 1168, Centre de St Jérôme, Avenue \\ Escadrille Normandie Niémen, 13397 Marseille, France
}

(Reçu le 27 avril 1989, révisé et accepté le 25 septembre 1989)

\begin{abstract}
Résumé. - On rappelle les équations de base de la méthode des Eléments de Frontière en diffusion thermique stationnaire et on montre en quoi cette méthode est bien adaptée pour traiter des problèmes inverses en conduction. Pour les problèmes inverses stationnaires, on décrit la formulation Eléments de Frontière en l'associant à une procédure numérique de régularisation. Deux exemples d'application sont présentés : l'estimation de coefficients de transfert à la périphérie d'un cylindre d'après des mesures de température au sein du matériau et l'estimation du flux surfacique appliqué à la face cachée d'un système à partir de mesures thermographiques infrarouges sur sa face visible.

Summary. - The basic equations of the Boundary Element Method in stationary thermal diffusion are reviewed and it is explained why this method is well adapted to inverse conduction. For stationary inverse problems the Boundary Element formulation using regularization numerical algorithms is described. Two applications are presented : the determination of the heat transfer coefficient around a cylinder from bulk temperature measurements and the determination of the surfacic flux acting on the hidden side of a thermal system by using infrared thermographic measurements on its visible side.
\end{abstract}

\section{Nomenclature.}

$A$ : matrice (Eq. (20))

$b: g / \lambda$

B : vecteur (Eq. (20))

$c$ : coefficient multiplicatif

$d$ : distance

$g$ : source de chaleur

$G$ : matrice (Eq. (6))

$H$ : matrice (Eq. (6))

$i$ : indice de point ou d'élément

$K$ : transformée de Kirchoff

$M$ : point de $\Gamma$ ou $\Omega$

$n$ : vecteur unitaire sortant normal à $\Gamma$ en $\mathrm{P}$

$N$ : nombre d'éléments

$N_{1}$ : nombre d'équations

$N_{2}$ : nombre d'inconnues

$\mathrm{P}$ : point de $\Gamma$

$p$ : densité de flux

$q: p / \lambda$

$q^{*}$ : fonction de pondération

$r$ : distance de $\mathbf{M}$ à $\mathbf{P}(\mathbf{r}=\mathbf{M P})$
$R$ : matrice de régularisation

$S$ : vecteur termes sources

$T$ : température

$\mathbf{T}$ : vecteur des températures

$T^{*}$ : fonction de Green

$V$ : matrice (Eq. (22))

$\mathbf{X}$ : vecteur des inconnues

' : exposant relatif aux points internes

\section{SYMBOLES GRECS}

$\nabla$ : opérateur gradient au point $\mathrm{P}$

$\lambda$ : conductivité

$\mu$ : coefficient de régularisation

$\Omega$ : domaine

$\Psi$ : transformée de Kirchoff de $T$

\section{Introduction.}

En modélisation, la résolution de «problèmes directs » permet de simuler des phénomènes physiques et si ces phénomènes sont régis par des équations aux dérivées partielles, différentes techni- 
ques numériques peuvent être utilisées: les plus connues sont les Eléments Finis et les Différences Finies et l'une des plus récentes est la méthode des Eléments de Frontière [1-3]. La résolution des problèmes directs nécessite la connaissance de conditions aux limites: dans le cas des transferts de chaleur par conduction auxquels nous nous intéressons ici, il s'agit de la température ou bien du flux surfacique sur l'ensemble du contour du domaine considéré. Beaucoup d'études industrielles ne nécessitent toutefois pas la résolution de problèmes directs mais de «problèmes inverses », afin par exemple de déterminer des températures ou flux de contour d'après des mesures internes au corps étudié $[4,5]$. Dans cet article nous présentons une approche basée sur une formulation de type intégrale de contour de l'équation de la chaleur, pour traiter des problèmes inverses en conduction. Ces problèmes sont en fait beaucoup plus difficiles que les problèmes directs, car «mal posés » au sens d'Hadamard [6].

Dans le paragraphe 2, on rappelle d'abord les équations de base de la méthode des Eléments de Frontière, en conduction stationnaire $2 \mathrm{D}$ et $3 \mathrm{D}$ (bi et tridimensionnelle), et dans le cas d'une diffusivité dépendant de la température. Le couplage entre les températures internes et les températures et flux surfaciques de contour assuré par ces équations intégrales, met clairement en évidence l'intérêt de la méthode pour les problèmes inverses en conduction. Après discrétisation spatiale, on obtient la forme discrète des équations, sous forme de relations matricielles entre températures et flux surfaciques des éléments de contour d'une part, et températures internes et champs de contour d'autre part.

Dans le paragraphe 3 on traite du problème inverse, c'est-à-dire de la détermination de champs de température et flux surfaciques de contour, connaissant par exemple un ensemble de températures internes au corps étudié. Alors que dans le cas du problème direct, on est toujours conduit à la résolution d'un système d'équations dont les nombres d'équations et d'inconnues sont égaux, dans le cas du problème inverse on obtient en général un système d'équations surdéterminé. Le problème inverse abordé étant mal posé, on fait appel aux techniques numériques de régularisation [7] avant de résoudre ce système d'équations au sens des moindres carrés.

Enfin, deux exemples d'application sont présentés dans le paragraphe 4. Le premier est relatif à la résolution du problème inverse posé en [8] pour la détermination des variations des coefficients de transfert thermique à la périphérie d'un cylindre dans un écoulement. Dans le second, on calcule le champ de flux appliqué sur la face cachée d'un système thermique d'après des mesures thermographiques infrarouges sur sa face visible; ce système est un barreau de section carrée percé en son centre.

\section{Equations de base.}

On rappelle les formulations continue et discrète de la méthode des Eléments de Frontière et l'on montre comment traiter de non-linéarités sur le contour ou sur le domaine, quand la conductivité varie avec la température.

2.1 Formulation CONTINUE. - Considérons un domaine $\Omega$ limité par son contour $\Gamma$. Dans le cas stationnaire linéaire, l'équation fondamentale des Eléments de Frontière s'établit en annulant l'intégrale du produit du résidu de l'équation de chaleur et d'une fonction de pondération $T^{*}$; après une double intégration de l'expression ainsi obtenue et le choix d'une fonction de Green pour $T^{*}$, on trouve que pour tout point $\mathrm{M}$ de $\Omega[1]$ :

$c T_{\mathrm{M}}+\int_{\Gamma} T q^{*} \mathrm{~d} \Gamma=\int_{\Gamma} q T^{*} \mathrm{~d} \Gamma+\int_{\Omega} b T^{*} \mathrm{~d} \Omega$

où :

$c=1$, si $\mathbf{M}$ appartient à $\Omega$ et n'appartient pas à $\Gamma$ $c=0,5$, si $\mathrm{M}$ appartient à $\Gamma$ et si $\Gamma$ n'est pas anguleux en $M$.

Dans cette équation intégrale $T^{*}(\mathrm{M}, \mathrm{P})$ est une fonction de Green et $q^{*}$ provient de sa dérivée ; leurs expressions dépendent de la dimension de l'espace du domaine $\Omega$, de la distance $r$ du point $M$ au point $\mathrm{P}$ de $\Gamma$ et du produit scalaire $d[9]$ :

- en 2D :

$$
\begin{gathered}
T^{*}=\frac{1}{2 \pi} \ln \left(\frac{1}{r}\right) \quad q^{*}=\nabla T \cdot \mathbf{n}=\frac{d}{2 \pi r^{2}} \\
d=-\mathbf{r} \cdot \mathbf{n}
\end{gathered}
$$

- en 3D:

$$
T^{*}=\frac{1}{4 \pi} \frac{1}{r} \quad q^{*}=\frac{d}{4 \pi r^{3}}
$$

En instationnaire une démarche similaire peut être suivie, en complétant la double intégration spatiale par une intégration sur le temps. Les équations alors obtenues sont notamment présentées en [3].

L'examen de la représentation intégrale (1) met clairement en évidence l'intérêt que peut présenter l'approche Eléments de Frontière pour inverser l'équation de la chaleur, puisqu'elle permet de coupler les variations d'une température interne (cas $c=1$ ) aux variations de la température et du flux sur le contour $\Gamma$.

2.2 Formulation DisCRÈTE. - Supposons dans un premier temps le terme source de chaleur nul. Dans ce cas n'apparaissent dans l'équation (1) que des intégrales de contour et seul le maillage de $\Gamma$ en «éléments de frontière » est donc nécessaire. La manière la plus simple de procéder est alors de 
supposer les températures et les flux uniformes sur chaque élément: $T_{j}$ et $q_{j}$ sur l'élément $\Gamma_{j}$. En introduisant les coefficients $H_{i j}$ et $G_{i j}$ tels que,

$$
H_{i j}=\int_{\Gamma_{j}} q^{*}\left(\mathrm{P} ; \mathrm{M}_{i}\right) \mathrm{d} \Gamma
$$

et

$$
G_{i j}=\int_{\Gamma_{j}} T^{*}\left(\mathrm{P} ; \mathrm{M}_{i}\right) \mathrm{d} \Gamma
$$

l'équation (1) exprimée pour l'élément de contour $i$ donne ( $N$ : nombre d'éléments de contour) :

$$
\frac{1}{2} T_{i}+\sum_{j=1}^{N} H_{i j} T_{j}=\sum_{j=1}^{N} G_{i j} q_{j}
$$

Cette expression peut être formulée pour les $N$ éléments de $\Gamma$. En introduisant alors les matrices $H$ et $G$ telles que ( $\delta_{i j}$ : symbole de Kronecker) :

$$
[H]_{i j}=H_{i j}+\frac{1}{2} \delta_{i j} \quad \text { et } \quad[G]_{i j}=G_{i j}
$$

on obtient le système d'équations algébriques :

$$
H \mathbf{T}=G \mathbf{Q} .
$$

Dans le cas d'un problème direct, la prise en compte des conditions aux limites conduit alors à distinguer les grandeurs connues des grandeurs inconnues :

- condition de Dirichlet sur $\Gamma_{i}: T_{i}=\mathrm{T}_{\mathrm{i}}$;

- condition de Neuman sur $\Gamma_{i}: q_{i}=\mathrm{q}_{\mathrm{i}}$;

- condition de Fourier sur $\Gamma_{i}: q_{i}=\alpha_{i} T_{i}+\beta_{i}$.

En groupant les grandeurs inconnues dans un vecteur $\mathbf{X}$, un arrangement de (7) conduit à formuler un système d'équations linéaires dont $\mathbf{X}$ est solution.

Une fois ce système résolu, on dispose de l'ensemble des températures et des flux $T_{i}$ et $q_{i}$. Il est alors possible de déterminer la température en tout point interne ; ainsi, pour un point interne $\mathbf{M}_{i}^{\prime}$, en introduisant les coefficients $H_{i j}^{\prime}$ et $G_{i j}^{\prime}$ définis comme en (4), avec $c=1$ dans (1) on a :

$$
T_{i}^{\prime}+\sum_{j=1}^{N} H_{i j}^{\prime} T_{j}=\sum_{j=1}^{N} G_{i j}^{\prime} q_{j}
$$

Si cette expression est formulée pour $N^{\prime}$ points internes, en introduisant les matrices $H^{\prime}$ et $G^{\prime}$ d'éléments $H_{i j}^{\prime}$ et $G_{i j}^{\prime}$ on obtient :

$$
\mathbf{T}^{\prime}=-H^{\prime} \mathbf{T}+G^{\prime} \mathbf{Q} .
$$

Le terme source de chaleur a précédemment été supposé nul. Dans le cas contraire apparaît simplement un terme source supplémentaire dans les équations (7) et (9). En notant $\mathbf{S}$ et $\mathbf{S}^{\prime}$ les vecteurs, respectivement associés aux points de contour et aux points internes, résultant de la prise en compte d'une source de chaleur, les équations (7) et (9) deviennent donc :

$$
\begin{gathered}
H \mathbf{T}=G \mathbf{Q}+\mathbf{S} \\
\mathbf{T}^{\prime}=-H^{\prime} \mathbf{T}+G^{\prime} \mathbf{Q}+\mathbf{S}^{\prime} .
\end{gathered}
$$

La difficulté est de calculer $\mathbf{S}$ et $\mathbf{S}^{\prime}$ sans recourir à un maillage du domaine $\Omega$, c'est-à-dire en n'utilisant que le maillage de $\Gamma[10]$.

2.3 EXTENSION AU CAS NON LINÉAIRE. - On envisage les cas de non-linéarités dues à une dépendance de la conductivité avec la température et à des conditions aux limites complexes.

$\mathrm{Si}$ la conductivité thermique est fonction de la température le problème devient non linéaire sur le domaine. On peut toutefois se ramener au cas linéaire par utilisation de la transformation de Kirchoff :

$$
\psi=K(T)=\int_{0}^{T} \lambda\left(T^{\prime}\right) \mathrm{d} T^{\prime} .
$$

L'identité :

$$
\nabla(\lambda \nabla T)=\Delta \Psi
$$

permet en effet une linéarisation rigoureuse de l'équation de la chaleur. Dans le cas stationnaire non linéaire, l'équation fondamentale s'écrit alors :

$$
c \Psi_{\mathrm{M}}+\int_{\Gamma} \Psi q^{*} \mathrm{~d} \Gamma=\int_{\Gamma} p T^{*} \mathrm{~d} \Gamma+\int_{\Omega} g T^{*} \mathrm{~d} \Omega .
$$

La discrétisation de cette équation s'effectue ensuite comme dans le cas linéaire, d'où des formes discrétisées similaires à (10) et (11).

La prise en compte de conditions aux limites non linéaires n'est pas plus complexe par Eléments de Frontière que par les méthodes numériques traditionnelles, car les variables sont précisément aux limites. Dans notre approche nous procédons par linéarisation locale, c'est-à-dire, après discrétisation, par des linéarisations propres à chacun des éléments de contour [10,11]. Pour une condition de type mixte on obtient ainsi, par linéarisation autour du cham $\Psi, \quad$ :

$$
p-p_{1}=\left(\frac{\mathrm{d} p}{\mathrm{~d} \psi}\right)_{\psi_{1}}\left(\psi-\psi_{1}\right)
$$

soit :

$$
p-p_{1}=\frac{1}{\lambda\left(T_{1}\right)}\left(\frac{\mathrm{d} p}{\mathrm{~d} T}\right)_{\mathrm{T}_{1}}\left(\psi-\psi_{1}\right) .
$$

Un processus itératif est ensuite utilisé : à chaque itération le problème est linéaire d'où le calcul d'un nouveau champ $\left(\Psi_{1}, p_{1}\right)$, jusqu'à stabilisation du résultat. 
Exemple : pertes radiatives ( $\varepsilon$ : facteur d'émission, $\sigma$ : constante de Stéfan) :

$$
p=\varepsilon \sigma T^{4}
$$

l'équation linéarisée s'écrit :

$$
p=\frac{4 \varepsilon \sigma T_{1}^{3}}{\lambda\left(T_{1}\right)}\left(\psi-\psi_{1}\right)+\varepsilon \sigma T_{1}^{4} .
$$

\section{Traitement du problème inverse.}

En utilisant les formulations discrètes précédemment établies, on montre comment résoudre les problèmes inverses de détermination de températures et de flux surfaciques de contour et l'on décrit la technique numérique de régularisation employée.

3.1 DÉTERMINATION DE CHAMPS DE TEMPÉRATURE ET DE FLUX SURFACIQUE DE CONTOUR. - Les équations (10) et (11) peuvent être récrites :

$$
\left[\begin{array}{c}
H \\
H^{\prime}
\end{array}\right] \mathbf{T}=\left[\begin{array}{c}
G \\
G^{\prime}
\end{array}\right] \mathbf{Q}+\left[\begin{array}{c}
\mathbf{S} \\
\mathbf{S}^{\prime}-\mathbf{T}^{\prime}
\end{array}\right]
$$

Supposons par exemple connues les températures internes et inconnus les champs de contour ; dans ce cas les grandeurs inconnues sont solution du système d'équations :

$$
\left[\begin{array}{l}
H-G \\
H^{\prime}-G^{\prime}
\end{array}\right]\left[\begin{array}{l}
\mathbf{T} \\
\mathbf{Q}
\end{array}\right]=\left[\begin{array}{c}
\mathbf{S} \\
\mathbf{S}^{\prime}-\mathbf{T}^{\prime}
\end{array}\right] .
$$

Ce système comporte $N+N^{\prime}$ équations et $2 N$ inconnues. Il est donc sous-déterminé et inexploitable si $N^{\prime}<N$, carré si $N=N^{\prime}$ et surdéterminé si $N^{\prime}>N$. Dans le cas monodimensionnel, pour lequel $N=2$, on retrouve que 2 températures internes sont nécessaires pour pouvoir traiter du problème inverse.

Avant de poursuivre le développement, on peut se placer dans un cas plus général que celui qui vient d'être envisagé. Comme schématisé figure 1, on peut supposer disposer en certaines portions de contour des classiques conditions aux limites (Neuman, Dirichlet et Fourier), connaître en d'autres points les champs de température et de flux et connaître également un ensemble de températures internes. Comme dans l'exemple précédent, il sera alors toujours possible de réarranger (18) afin de grouper les grandeurs inconnues dans un vecteur $\mathbf{X}$ et de constituer un système d'équations algébriques :

$$
A \mathbf{X}=\mathbf{B} \text {. }
$$

Soit $N_{1}=N+N^{\prime}$ le nombre d'équations et $N_{2}$ le nombre d'inconnues de ce système. Si $N_{1}<N_{2}$ le système est inexploitable car sous-déterminé, si $N_{1}=N_{2}$ on peut calculer $\mathbf{X}$ et si $N_{1}>N_{2}$ le système est surdéterminé. Cette dernière hypothèse est celle habituellement rencontrée ; dans ce cas, si

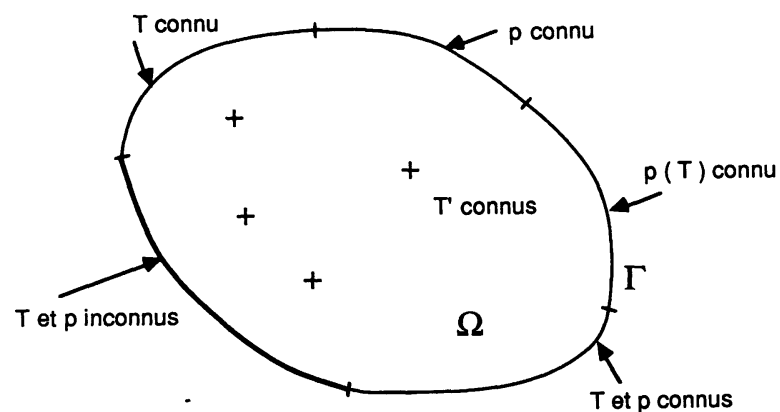

Fig. 1. - Problème inverse considéré : calcul des flux surfaciques et températures sur une portion de contour disposant (i) de classiques conditions aux limites et (ii) de températures internes et/ou de flux surfaciques et températures de contour.

[Inverse problem on consideration : calculation of temperatures and surfacic fluxes on a part of the boundary knowing (i) classical boundary conditions and (ii) internal temperatures and/or temperatures and surfacic fluxes on the boundary.]

les relations de (20) sont linéairement indépendantes on doit envisager de trouver une solution au sens de la minimisation d'une norme de l'écart entre $A \mathbf{X}$ et $\mathbf{B}$.

$$
\hat{\mathbf{X}}=\min \|A \mathbf{X}-\mathbf{B}\| .
$$

Des considérations statistiques conduisent à retenir une norme quadratique [12]: en supposant l'écart entre $A \mathbf{X}$ et $\mathbf{B}$ aléatoire gaussien centré de matrice de variance-covariance connue, d'inverse $V$ ( $V$ symétrique définie positive), on choisira :

$$
\|A \mathbf{X}-\mathbf{B}\|^{2}=(A \mathbf{X}-\mathbf{B})^{T} V(A X-\mathbf{B}) .
$$

On obtient alors pour $\mathbf{X}$ l'estimateur optimal de Markov, qui est solution du système de $N_{2}$ équations à $N_{2}$ inconnues :

$$
A^{T} V A \mathbf{X}=A^{T} V \mathbf{B} .
$$

En pratique, l'estimateur de Markov n'a qu'un intérêt théorique, car la matrice $V$ est généralement inconnue ; en posant $V=1$ (1: matrice identité) dans (22) et (23), on a simplement la solution au sens des moindres carrés qui suppose la matrice de variance-covariance diagonale (pas de corrélation spatiale dans les écarts) et d'éléments égaux (amplitude des écarts similaires).

\subsection{RÉGULARISATION DU PROBLÈME D'INVERSION.} - La difficulté en général rencontrée est la grande sensibilité aux bruits additifs sur les mesures utilisées. Cette difficulté est classique en inversion de l'équation de la chaleur et plus généralement en déconvolution ; elle est due au fait que le problème d'inversion est « mal posé ». Cela se traduit, en (23), 
par un mauvais conditionnement de la matrice à inverser. Les solutions numériques à ce type de problème relèvent des techniques de régularisation [7].

Supposons par exemple que $\mathbf{X}$ représente, sous forme discrétisée, les variations d'un flux surfacique $x$ sur un élément de contour $\Gamma_{x}$. On pourra alors régulariser la solution en adjoignant à la forme quadratique (22) un terme régularisateur : habituellement on choisit le produit d'un coefficient de régularisation $\mu$ et de la norme Euclidienne de $x$ (régularisation d'ordre 0 ), ou de sa dérivée première (régularisation d'ordre 1), de sa dérivée seconde (régularisation d'ordre 2) etc... ou encore d'une combinaison de ces normes. Sous forme discrétisée, et d'une façon générale, cela reviendra à substituer à (21) le problème d'optimisation :

$$
\hat{\mathbf{X}}=\min \left(\|A \mathbf{X}-\mathbf{B}\|^{2}+\mu\|R \mathbf{X}\|^{2}\right)
$$

avec $R$ une matrice fonction de l'ordre choisi pour la régularisation, de l'arrangement des composantes de $\mathbf{X}$ etc...

La solution de ce nouveau problème vérifie le système de $N_{2}$ équations à $N_{2}$ inconnues :

$$
\left(A^{T} V A+\mu R^{T} R\right) \mathbf{X}=A^{T} V \mathbf{B} .
$$

Suivant la valeur du coefficient de régularisation $\mu$, la solution sera plus ou moins régulière.

3.3 CONSIDÉRATIONS NUMÉRIQUES. - Les développements présentés ci-dessous ont permis d'étendre le logiciel Eléments de Frontière développé dans notre laboratoire $[3,10,11]$; ce logiciel comporte maintenant un module susceptible de traiter en stationnaire le problème inverse schématisé figure 1 : détermination de champs de température et flux surfacique sur des portions de contour connaissant un ensemble de températures internes et/ou des champs de température et de flux surfacique sur d'autres portions de contour.

En pratique, deux types supplémentaires de conditions aux limites peuvent être considérés dans le module problème inverse :

- température et flux surfacique connus ;

et les températures internes constituent un ensemble de données complémentaires.

Pour la régularisation, on peut travailler avec les températures ou/et avec les flux en manipulant des matrices de régularisation, dont seront issues $R$ (cf. (24)), et des coefficients de régularisation $\mu$ relatifs aux températures et aux flux. Cette possibilité est un avantage spécifique à la méthode des Eléments de Frontière dont la formulation (cf. (1)) fait apparaître de manière symétrique et explicite les températures et les flux de contour.
Comme les modules numériques relatifs aux problèmes directs, le module problème inverse stationnaire est utilisable dans les cas suivants :

- géométries bi et tridimensionnelles ;

- conditions aux limites non linéaires ;

- conductivité fonction de la température ;

- domaine d'étude composé de plusieurs régions de conductivités thermiques différentes;

- sources de chaleur ponctuelles ou dérivant d'un potentiel [10].

Le module utilisable en transitoire est en cours de développement.

\section{Exemples d'application}

On présente dans ce paragraphe deux exemples d'application du logiciel développé.

\subsection{COEFFICIENT DE TRANSFERT LOCAL À LA PÉRI-} PHÉRIE D'UN CYLINDRE DANS UN ÉCOULEMENT UNIDIRECTIONNEL PAR MESURE DE TEMPÉRATURES INTERNES. - Comme présenté en [8] la mesure de températures dans un cylindre peut permettre, par inversion de l'équation de la chaleur, la détermination du coefficient de transfert à la périphérie de ce cylindre (Fig. 2). Connaissant le champ de température sur la face interne et un ensemble de températures dans le cylindre (correspondant en fait à un ensemble d'expérimentations), on calcule les champs de température et de flux surfacique périphériques. Connaissant les variations du flux surfacique périphérique et la température de l'environnement, on en déduit le coefficient de transfert local en fonction de l'angle polaire.

Pour traiter ce problème bidimensionnel, symétrique par rapport à la direction de l'écoulement, le

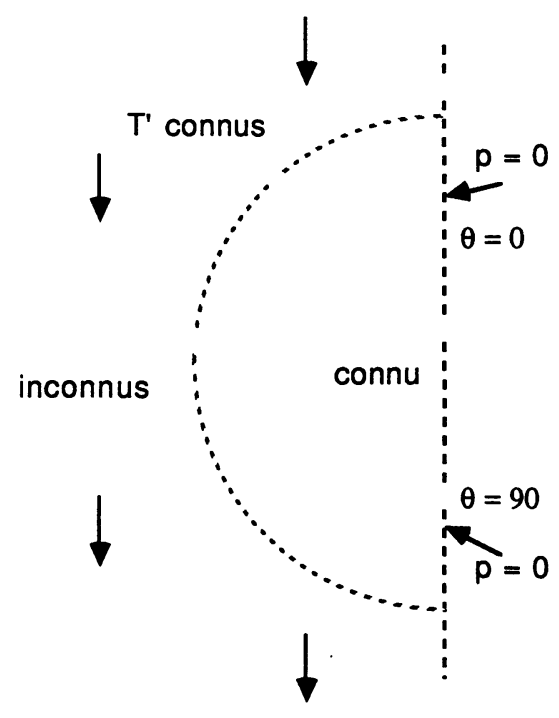

Fig. 2. - Expérimentation 1: schéma du dispositif.

[Experiment 1 : dispositive design.] 
contour du domaine d'étude a été discrétisé en $N=52$ éléments. Sur le cercle interne (rayon $8 \mathrm{~mm}$ ), approximé par 18 éléments quadratiques, la température est connue d'où une condition de Dirichlet; aux plans de symétrie, définis par $2 * 8$ éléments linéaires, le flux surfacique est nul d'où une condition de Neuman homogène; sur le cercle externe (rayon $16 \mathrm{~mm}$ ), approximé par 18 éléments quadratiques, la température et le flux surfacique sont inconnus. Dans le cylindre on connaît 18 températures régulièrement distribuées sur un cercle (rayon $14 \mathrm{~mm}$ ). Dans le cas de cette étude le système (20) est alors carré $(70 * 70)$.

D'après des mesures faites au LEMTA (Nancy) on présente figure 3 la courbe de variation angulaire

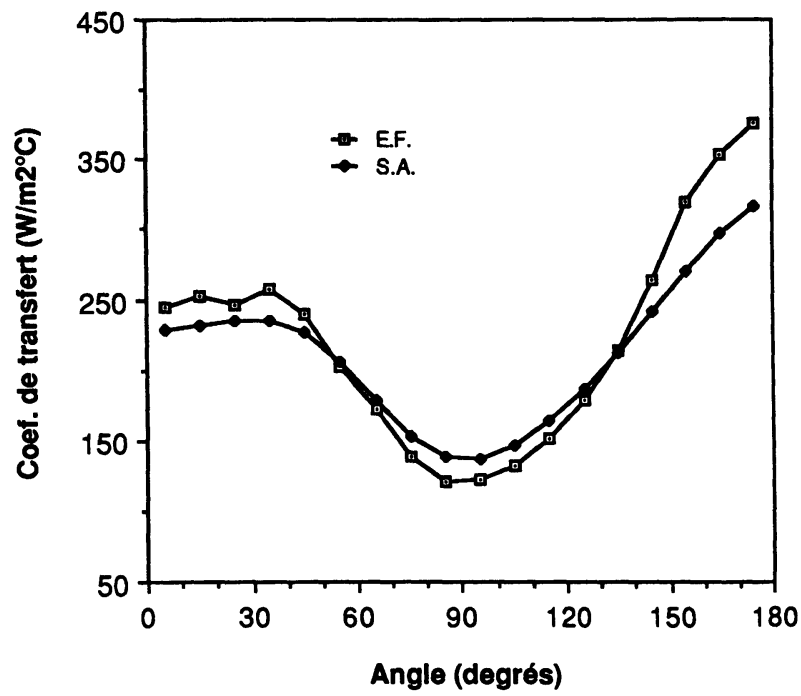

Fig. 3. - Expérimentation 1 : résultat Eléments de Frontière (E.F.) et Semi-Analytique (S.A.).

[Experiment 1: Boundary Elements (E.F.) and Semi Analytical (S.A.) results.]

du coefficient de transfert calculée par (i) Eléments de Frontière et par (ii) voie semi-analytique [8] (approche rendue possible par la simplicité de la géométrie). Ces variations ont été calculées en utilisant une régularisation d'ordre 2 sur les flux périphériques (cf. 3.2). Figure 4 on met en évidence l'influence de la régularisation en présentant les résultats obtenus par Eléments de Frontière pour diverses valeurs du coefficient de régularisation $\mu$.

\subsection{FLUX SURFACIQUE APPLIQUÉ À LA FACE NON} VISIBLE D'UN SYSTÈME PAR MESURE THERMOGRAPHIQUE I-R SUR SA FACE VISIBLE. - La thermographie infrarouge ne permettant d'accéder qu'à des champs de température superficielle, la détermination des causes des phénomènes observés nécessite en général la résolution de problèmes inverses (voir notamment [13, 14] en mécanique du solide).

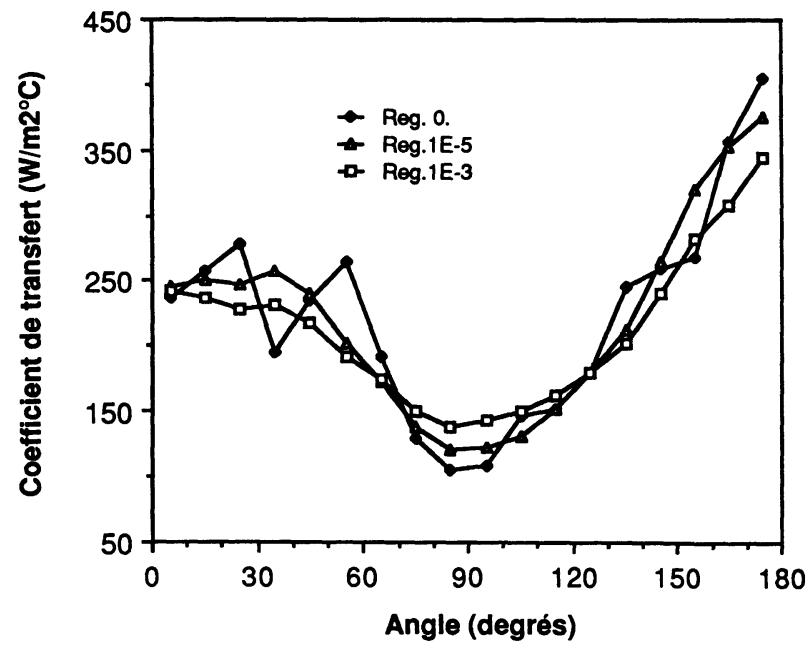

Fig. 4. - Expérimentation 1 : résultat Eléments de Frontière pour différentes valeurs du coefficient de régularisation.

[Experiment 1: Boundary Elements results for some values of the regularization coefficient.]

Connaissant un champ de température superficielle, l'équation de transfert thermique à l'interface et la température de l'environnement, on dispose des champs de température et de flux. Si ces champs résultent de flux appliqués à une face cachée du système thermique étudié, on peut alors envisager de les déterminer par résolution d'un problème inverse.

Pour vérifier la validité de cette approche nous avons mis en place l'expérimentation schématisée figure 5 ; il s'agit d'un barreau $(50 * 50 * 400 \mathrm{~mm})$ de plexiglas percé en son centre $(\varnothing 30 \mathrm{~mm})$ et à l'intérieur duquel sont placées deux résistances chauffantes et de l'isolant : chaque résistance est consituée d'un film chauffant occupant un quart de cercle. Les transferts conductifs sont bidimensionnels et la symétrie du système est telle que l'observation d'une face du barreau est suffisante pour résoudre le problème inverse.

Les calculs ont été menés par Eléments de Frontière et par «superposition": connaissant les champs de température obtenus lorsque des flux unitaires sont indépendamment appliqués sur les génératrices du cylindre interne au barreau, on peut, sous l'hypothèse de linéarité, utiliser le principe de superposition et en déduire le champ de température associé à toute distribution de flux. Cette méthode permet également l'inversion de l'équation de la chaleur [4] moyennant une régularisation spatiale. Elle nécessite le calcul préliminaire de l'ensemble des réponses à une entrée unitaire et n'est pas applicable dans le cas de conditions aux limites non linéaires.

Compte tenu de la symétrie du système, le domaine d'étude à considérer correspond à un quart 


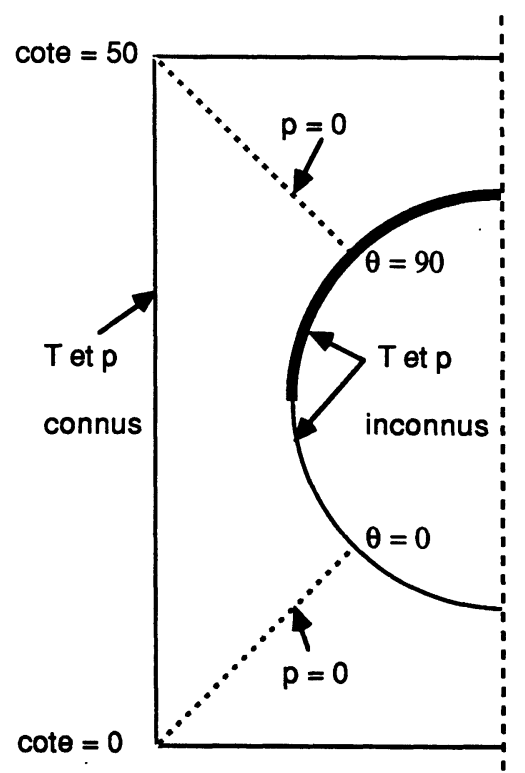

Fig. 5. - Expérimentation 2: schéma du dispositif (film chauffant en gras).

[Experiment 2: dispositive design (heating film in bold).]

de la section du barreau. La discrétisation est de $N=44$ éléments : sur la face filmée (largeur $50 \mathrm{~mm}$ ), définie par 18 éléments linéaires, on considère connus les champs de température et de flux ; sur le cylindre interne (rayon $15 \mathrm{~mm}$ ), approximé par 10 éléments quadratiques, les champs sont inconnus ; aux plans de symétrie, définis par $2 * 8$ éléments linéaires, on a des conditions de Neuman homogènes.

L'expérimentation a été menée en utilisant un dispositif d'acquisition et de numérisation d'images thermographiques infrarouges en temps réel [15]. Le profil thermique ainsi obtenu sur une face du

16

14

12

$\underset{\leftarrow}{\check{L}}$

10

8

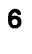

$$
\begin{array}{ll}
20 \quad 30 & 30 \\
\text { Cote }(\mathrm{mm})
\end{array}
$$

Fig. 6. - Expérimentation 2: profil thermique sur une face du barreau.

[Experiment 2 : thermal profile along the bar side.] barreau est présenté figure 6. Pour déterminer le profil de flux surfacique, nécessaire au calcul inverse, on a supposé que le transfert de chaleur était proportionnel à l'écart de température avec la température ambiante. La difficulté provient alors de l'estimation du coefficient de transfert; nous avons retenu celui conduisant à une minimisation des minima de la fonctionnelle définie en (24).

Les résultats obtenus par Eléments de Frontière et Superposition apparaissent figure 7, sous la forme

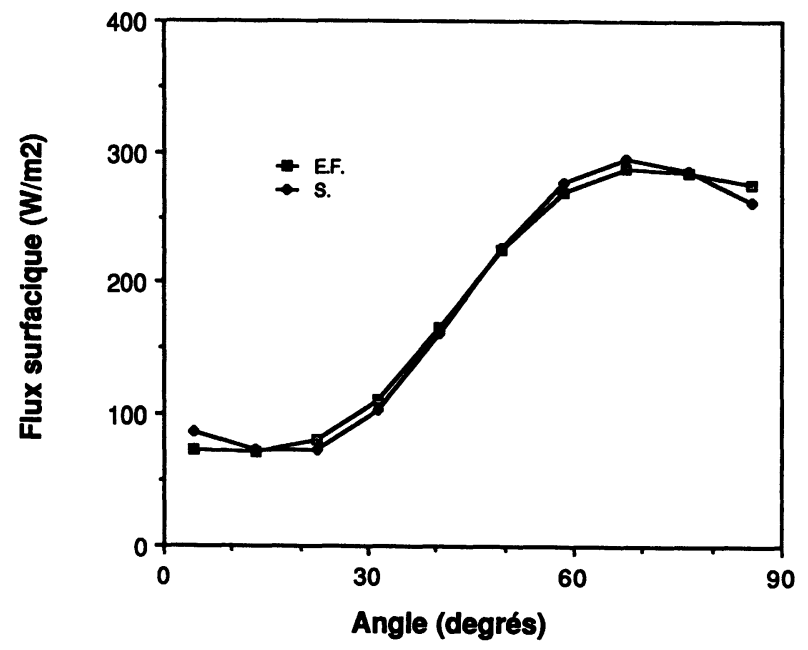

Fig. 7. - Expérimentation 2 : résultat Eléments de Frontière (E.F.) et par Superposition (S.).

[Experiment 2 : Boundary Elements (E.F.) and Superposition (S.) results.]

des variations du flux surfacique appliqué à l'intérieur du cylindre en fonction de l'angle polaire, et on montre figure 8 l'influence de la régularisation. Ces résultats prouvent la bonne cohérence entre les méthodes et doivent donc être considérés comme encourageants. La présence de la résistance chauffante, entre $45^{\circ}$ et $90^{\circ}$, n'est toutefois pas clairement mise en évidence. Cela atteste de l'intérêt des progrès technologiques et expérimentaux permettant une amélioration de la mesure, pour le traitement des problèmes inverses à partir de données thermo-

\section{Conclusion.}

La méthode des Eléments de Frontière est bien adaptée à l'intégration numérique de l'équation de la chaleur et plus généralement aux problèmes pour lesquels un champ dérive d'un potentiel (électrostatique, écoulement irrotationnel, transfert de masse par diffusion...) ; nous avons montré ici qu'elle était également adaptée au traitement des problèmes inverses de cette nature, en fournissant notamment 


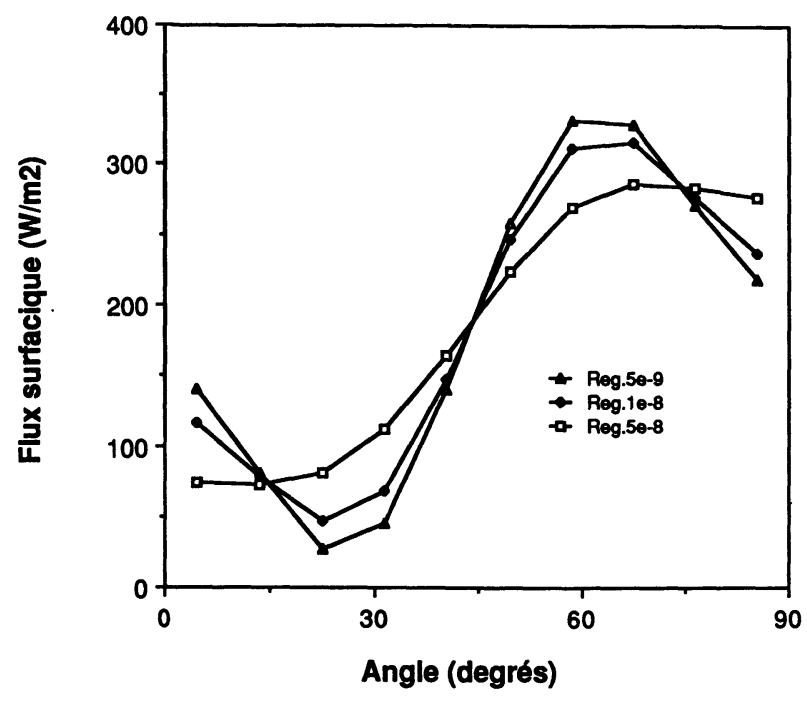

Fig. 8. - Expérimentation 2 : résultat Eléments de Frontière pour différentes valeurs du coefficient de régularisation.

[Experiment 2: Boundary Elements results for some values of the regularization coefficient.]

des équations de couplage entre variables internes et champs de contour. Par rapport aux approches numériques traditionnelles, la méthode des Eléments de Frontière a des propriétés intéressantes : diminution d'une unité de la dimension du problème (3D en 2D, 2D en 1D) ; - précision de la solution ; - possibilité de traiter simplement des milieux non bornés ; - possibilité de tenir compte simplement de singularités. Ces propriétés, intrinsèques à la méthode, se retrouvent en problèmes inverses comme en problèmes directs, stationnaires comme transitoires. Un module numérique utilisable en transitoire est ainsi en cours de développement.
Après avoir rappelé les équations fondamentales de la méthode des Eléments de Frontière en formulations continue et discrète, nous avons montré comment traiter des problèmes inverses consistant à déterminer des champs de contour à partir d'informations complémentaires, températures internes par exemple. L'approche proposée conduit à résoudre un problème d'optimisation quadratique pour lequel on dispose d'une solution explicite. Dans le cas de conditions aux limites non linéaires, on procède par itérations et si la conductivité dépend de la température, l'équation de la chaleur est préalablement linéarisée par utilisation de la transformation de Kirchoff. Les solutions aux problèmes inverses étant très sensibles aux erreurs de mesure, des procédures numériques de régularisation sont nécessaires à l'obtention de résultats satisfaisants.

En dernier lieu deux exemples d'application ont été décrits. Le premier, relatif au calcul des variations d'un coefficient de transfert de chaleur à la périphérie d'un cylindre, nous a permis de retrouver des résultats précédemment présentés [8] ; un tel calcul pourrait maintenant être mené dans des cas de géométries complexes. La seconde application a consisté à estimer un flux surfacique sur la face cachée d'un système thermique d'après des mesures thermographiques sur la face visible, la difficulté rencontrée ici réside en fait dans la bonne connaissance du champ de flux surfacique sur la face visible, nécessaire au traitement du problème inverse. Les bons résultats obtenus accréditent l'adéquation de la méthode des Eléments de Frontière au traitement des problèmes inverses, qui sont cependant par nature difficiles et qui doivent donc être abordés en disposant de mesures de qualité en des endroits soigneusement choisis, afin essentiellement que la sensibilité de la solution aux variations des mesures ne soit pas négligeable.

\section{Bibliographie}

[1] Brebila C. A., The Boundary Element Method for engineers (Pentech Press) 1978.

[2] Brebbia C. A., Telles J. C. F., Wrobel L. C., Boundary Element Techniques, Theory and application in engineering (Springer-Verlag) 1984.

[3] Pasquetti R., Caruso A., Brebbia C. A., Méthode des Eléments de Frontière : résolutions numériques des problèmes de diffusion thermique, Rev. Gen. Th. (Avril 1989).

[4] Beck J. V., Blackwell B., Saint-Clair C. R., Inverse heat conduction, ill posed problems, Wiley Interscience (1985).

[5] Raynaud M., BRANSIER J., A new finite difference method for the non linear inverse heat conduction problem, Heat Transfer $9 n^{\circ} 1$ p. 27.
[6] Hadamard J., Le problème de Cauchy (Hermann, Paris) 1932.

[7] Tikhonov A., Arsenine V., Méthode de résolution des problèmes mal posés (Editions de Moscou) 1976.

[8] Maillet D., Degiovanni A., Méthode analytique de conduction inverse appliquée à la mesure d'un coefficient de transfert local sur un cylindre en convection forcée, Rev. Ph. Appl. à paraître.

[9] OzIsıK N. N., Heat conduction (John Wiley \& Sons) 1980.

[10] Pasquetri R., A Boundary Element software for the numerical integration of the heat conduction equation, INME symposium : numerical 
methods in engineering (EPFL Lausanne) septembre 1989.

[11] Pasquetti R., Caruso A., A new software for the modelization of transient and non linear thermal diffusion, Conférence BEM10 (Southampton) 6 au 9-9-88.

[12] De Larminat P., Thomas Y., Automatique des systèmes linéaires, Tome 2 : Identification (Flammarion Sciences) 1977.
[13] NAYrolles B. et al., Téléthermographie infrarouge et mécanique des structures, Int. J. Engng. Sci. 19 (1981) 929-947.

[14] Bouc R., Nayrolles B., Méthodes et résultats en thermographie infrarouge des solides, J. Méc. Théor. Appl. 4 (1985) 27-58.

[15] Guillemant P., Papini F. et al., Une évolution expérimentale dans la restitution des champs de température, Colloque visualisation et traitement d'images (Belfort) 18 au 20-5-1988. 